\title{
Questes
}

vestes Revue pluridisciplinaire d'études médiévales

12 | 2007

La faim et l'appétit

\section{Les envies de poisson dans la littérature médiévale}

\section{Cécile Le Cornec}

\section{(2) OpenEdition \\ Journals}

Édition électronique

URL : http://journals.openedition.org/questes/2704

DOI : 10.4000/questes. 2704

ISSN : 2109-9472

Éditeur

Les Amis de Questes

Édition imprimée

Date de publication : 15 juin 2007

Pagination : 11-22

ISSN : 2102-7188

\section{Référence électronique}

Cécile Le Cornec, «Les envies de poisson dans la littérature médiévale », Questes [En ligne], 12 | 2007 mis en ligne le 15 janvier 2014, consulté le 30 avril 2019. URL : http://journals.openedition.org/ questes/2704; DOI : 10.4000/questes.2704 


\section{Les envies de poisson dans la littérature médiévale}

\section{Cécile Le CORNEC}

Les personnages de la littérature médiévale, lorsqu'ils ont faim, rêvent souvent de poissons. Ce n'est certes pas le seul aliment qui excite leur appétit, mais il occupe une place privilégiée dans l'imagination des affamés et des gourmands. Or cet appétit pour le poisson peut sembler paradoxal. On considère habituellement les hommes du Moyen Âge comme de grands amateurs de viande, non sans raison. De plus, le poisson est à cette époque la nourriture des jours maigres par excellence, « la pièce essentielle de l'abstinence chrétienne » ${ }^{1}$. C'est un aliment imposé par l'Église pendant au moins un tiers de l'année et sa consommation est nettement associée aux périodes de pénitence ${ }^{2}$. Thomas d'Aquin justifie ainsi l'abstinence chrétienne dans sa Somme théologique: «Le jeûne a été institué par l'Église pour réprimer la convoitise des plaisirs du toucher qui ont pour objet la nourriture et la volupté. L'abstinence doit donc porter sur les aliments les plus délectables et les plus excitants. Telle est la chair des quadrupèdes et des oiseaux, tels aussi le laitage et les œufs. $»^{3}$ Le poisson, froid et humide selon les principes de la médecine galénique, est autorisé parce qu'il se distingue de ces «composés organiques plus rapprochés du corps humain, [...] plus agréables et plus nourrissants, plus capables aussi d'alimenter les sources de la luxure $»^{4}$. Contrairement aux autres aliments d'origine animale,

${ }^{1}$ Bruno LaURIOUX, Manger au Moyen Âge, Paris, Hachette Littératures, 2002, p. 104.

${ }^{2}$ Il reste difficile d'évaluer précisément le nombre de jours de jeûne car il varie selon les périodes et les régions. Il n'existe pas encore de synthèse à ce sujet. Pour une évaluation, voir Bruno LAURIOUX, op. cit., p. 105-113.

${ }^{3}$ Thomas D'Aquin, Somme théologique, trad. Jean-Dominique Folghera, Paris, Desclée et Cie, 1928, p. 144, cité par Carole LAMBERT, « La Nourriture comme signe de distinction religieuse et sociale de Thomas d'Aquin à Érasme ", Hérésis, Centre d'études cathares, 26-27 (1996), p. 101-102.

${ }^{4}$ Ibid. 
le poisson est donc supposé ne pas exciter les appétits charnels. Pourtant dans les textes littéraires, il se place du côté du plaisir des sens et connote le luxe et l'abondance. Anita Guerreau-Jalabert l'a souligné à propos de la matière arthurienne ${ }^{5}$, mais cette observation se vérifie quel que soit le genre des textes. Même dans la Bataille de Caresme et de Charnage, où le premier apparaît comme un triste sire, le conflit est déclenché par la jalousie de Charnage parce que dans certaines cours on lui préfère Carême, non pour lui-même, mais pour certains de ses gens particulièrement valeureux, les poissons frais ${ }^{6}$. Jusqu'à la seconde moitié du $\mathrm{XIV}^{\mathrm{e}}$ siècle, le poisson n'apparaît jamais dans la littérature comme un substitut décevant à la viande, mais au contraire comme un aliment qui suscite plus qu'un autre les appétits. Nous nous intéresserons dans un premier temps à ces envies de poisson et nous verrons qu'elles portent le plus souvent sur quelques espèces prestigieuses. Néanmoins, des poissons très courants, comme le hareng et l'anguille, sont aussi des objets de convoitise, plus spécialement dans les œuvres comiques. Nous nous interrogerons donc ensuite sur les connotations particulières attachées à ces deux espèces dans le fameux épisode du vol des poissons de la branche III (éd. Martin) du Roman de Renart.

\section{Les poissons de luxe}

a. Dire la faim : des poissons à foison

La plainte de la jeune comtesse, héroïne du Roman du comte d'Anjou, montre à quel point les poissons peuvent susciter le désir chez ceux qui ont faim. Dans ce roman écrit par Jean Maillart en 1316, qui reprend le canevas du conte de la « fille sans mains », la jeune fille doit fuir la folie incestueuse de son père,

\footnotetext{
${ }_{5}^{5}$ Anita Guerreau-JALABERT, «Aliments symboliques et symbolique de la table dans les romans arthuriens (XII ${ }^{\mathrm{e}}$-XIII ${ }^{\mathrm{e}}$ siècles) », Annales ESC, $\underline{3}_{2}$ (mai-juin 1992), p. 561-594.

${ }^{6}$ La Bataille de Caresme et de Charnage, éd. Grégoire LOZINSKI, Paris, Champion, 1933 (Bibliothèque de l'École des Hautes Études, 262), v. 77-81 : «Li Biauvoisin et li François / Et la gent devers Orlenois / Aiment assez mieus le poisson / Qu'il ne feroient venoison / Ne bone char de buef as aus. »
} 
le comte d'Anjou, qui veut l'épouser. Peu de temps après son départ, elle rencontre dans la forêt une pauvre femme qui lui propose du pain dur, noir et moisi. Elle sera contrainte de s'en contenter, mais auparavant elle prononce une longue plainte de 58 vers où elle regrette l'abondance et la finesse des mets qu'elle avait l'habitude de consommer chez son père'. La faim est dite paradoxalement par l'abondance des mets nommés. La comtesse se souvient d'abord des viandes (les volailles et le gibier, qui occupent 8 vers) puis vient le tour des poissons: en 28 vers sont mentionnées 31 espèces ainsi que des préparations variées, souvent raffinées ${ }^{8}$. Suivent les pâtisseries (5 vers) et les vins (11 vers). Sur les 52 vers qui énumèrent des mets particuliers, plus de la moitié est consacrée aux seuls poissons, qui apparaissent ainsi comme la nourriture la plus regrettée.

On peut lire cette litanie de la faim comme une digression plus ou moins gratuite qui donnerait à l'auteur l'occasion de déployer sa virtuosité et de solliciter l'appétit du lecteur, de faire se rencontrer le plaisir des mots et celui des mets. Cette interprétation n'est pas à exclure, d'autant plus que l'on retrouve nettement dans cette liste les espèces et les préparations présentes dans les livres de cuisine. La comtesse fait d'ailleurs directement allusion aux « cuisiniers qui connaissent les recettes $\gg{ }^{9}$. On peut alors, comme le fait Bruno Laurioux, lire ce passage comme un témoignage de l'émergence d'un discours gastronomique à partir du XIV ${ }^{e}$ siècle $^{10}$. Toutefois, si l'on replace l'extrait dans l'économie générale du récit, on peut aussi en proposer une interprétation plus morale. C'est ce que montre Madeleine Jeay dans Le Commerce des mots ${ }^{11}$, où elle s'interroge sur le sens et l'enjeu des listes de toute sorte insérées dans les textes littéraires. Elle rappelle que cet épisode se situe au tout début du long parcours de la

\footnotetext{
${ }^{7}$ Le passage en question est reproduit en annexe du présent volume.

${ }^{8}$ Le Roman du comte d'Anjou, éd. Mario RoQuES, Paris, Champion, 1964, v. 1116-1143.

${ }^{9}$ Ibid., v. 1141-1142 : « Des keus qui sevent lez sentances/ De l'atorner. »

${ }^{10}$ Bruno LAURIOUX, Manger au Moyen Âge, op. cit., p. 146-151. Voir aussi l'entretien avec Bruno Laurioux dans le présent volume.

${ }^{11}$ Madeleine JEAY, Le Commerce des mots. L'usage des listes dans la littérature médiévale (XII ${ }^{e}-X V^{e}$ siècles), Genève, Droz, 2006, p. 263-267.
} 
comtesse puis de son époux, le comte de Bourges. Dans la suite du roman, les deux protagonistes seront amenés à découvrir les vertus de l'humilité et de la charité. À la lumière des épreuves rédemptrices qui vont suivre, la liste des mets apparaît comme une manifestation de luxe dérisoire et vaine, marque de la corruption qui règne à la cour du comte d'Anjou. Dans l'interpolation contemporaine du Roman de Fauvel, Chaillou de Pestain, qui insère cette même liste presque inchangée dans le récit des noces de Fauvel, le roi âne, avec Vaine Gloire, explicite d'ailleurs sa dimension morale: elle est l'occasion d'une diatribe contre la « lescherie » et «l'ivresse », les vices de gloutonnerie.

Quelle que soit l'intention - morale ou non - de l'auteur, le poisson, se présente dans ces textes comme le plat favori des gourmands ou des gourmets. Les poissons nommés sont exceptionnels par leur qualité et leur quantité. S'ils occupent dans cette énumération une place si importante, c'est qu'ils suggèrent mieux que tout autre aliment le luxe, l'abondance et le raffinement.

\section{b. Poissons de rêve dans les œuvres comiques}

Cette liste est aussi dans une certaine mesure un développement des courtes énumérations stéréotypées de deux à quatre espèces, courantes dans les romans de chevalerie des XII ${ }^{\mathrm{e}}$ et XIII ${ }^{\mathrm{e}}$ siècles. Il s'agit d'un cliché stylistique qui permet d'indiquer qu'un repas est merveilleusement riche. Les $l u z$, lamproies et saumons ne sont peut-être pas dans le Graal, si l'on en croit l'ermite du roman de Chrétien de Troyes, mais ce sont de grands classiques des banquets romanesques. Il s'agit en général d'espèces connues dans les coutumes ou les livres de compte sous le nom de «poissons royaux » (d'après le latin juridique pisces regales): on en fait don aux grands personnages. Ces poissons extraordinaires, réservés aux nobles, sont courants aux tables des chevaliers, habitués aux nourritures merveilleuses.

En revanche, ils restent inaccessibles aux personnages des fabliaux ou du Roman de Renart qui se contentent d'en rêver. On les retrouve par exemple dans 
le «Fabliau de Cocagne ». Le jongleur, envoyé par le pape en pénitence dans ce lieu utopique entame ainsi sa description :
Li païs a a non Cocaigne ;
Qui plus i dort, plus i gaaigne :
Cil qui dort jusqu'à mïedi
Gaaigne .v. sols et demi.
De bars, de saumons et d'aloses
Sont toutes les mesons encloses ;
Li chevron i sont d'esturjons,
Les couvertures de bacons,
Et les lates sont de saussices. ${ }^{12}$

La description de cet ailleurs merveilleux s'ouvre par la mention de quatre espèces réputées, traditionnellement présentes dans les listes de poissons prestigieux. Les poissons royaux trouvent naturellement leur place dans ce lieu où se déploient les fantasmes les plus débridés, dans ce paradis perdu qui devient, dans l'espace de la parole, le réservoir des désirs inassouvis et des plaisirs hyperboliques.

Renart le rusé saura utiliser efficacement ce fantasme du pays de Cocagne. Pour persuader Isengrin de le rejoindre au fond du puits, le goupil lui fait miroiter l'accès à un lieu d'abondance où il trouvera tous les poissons qu'il désire. Renart a choisi l'appât le plus propre à allécher le loup en précisant : « Ou lus ou troites ou saumons ${ }^{13}$ ». Comme les animaux du Roman de Renart, certains personnages de fabliaux conservent l'espoir de posséder l'un de ces aliments si précieux. Mais leur attente sera presque toujours déçue. Ils doivent se contenter de maquereaux, de harengs ou, dans le meilleur des cas, de plies ${ }^{14}$ ou

\footnotetext{
${ }^{12}$ «Le Fabliau de Cocagne », éd. V. NÄÄNÄNEN, Neuphilologische Mitteilungen, 48 (1947), p. 22, v. 27-35.

${ }^{13}$ Le Roman de Renart, éd. Jean Dufournet et Andrée MÉLINE, Paris, Flammarion, 1985, vol. 1 (coll. GF 418), branche VI, p. 434, v. 622.

${ }^{14}$ "Les Trois aveugles de Compiègne », Nouveau Recueil Complet des Fabliaux, éd. Willem Noomen et Nico van den BoogaARD, Assen (Pays-Bas), Van Gorcum, 1984, tome II, 9, p. 178 , v. 100 .
} 
d'anguilles ${ }^{15}$. Seul l'exceptionnel Trubert parvient à réaliser ce rêve de tout personnage de fabliau: au terme de son long parcours, dans la dernière historiette, il prend place à la table du roi Golïas qui vient de l'épouser. Avant une nuit de noce mouvementée, Trubert a l'occasion de déguster, en plus des volailles les plus fines, un poisson rare, royal et à certains égards divins : l'esturgeon ${ }^{16}$.

Les frustrations des périodes maigres ne sont sans doute pas étrangères à cet attrait pour les poissons royaux exprimé par les textes littéraires. Ces espèces prestigieuses mettent en jeu une hiérarchie sociale. Or, pendant les jours maigres, les écarts entre les plus riches et le reste de la population se creusent. Seuls quelques privilégiés bénéficient d'une alimentation variée et apprêtée avec soin, alors que la majorité se contente de hareng salé. On imagine combien les désirs de nourriture devaient être exacerbés lorsque plusieurs jours maigres se succédaient, notamment pendant les quarante jours du Carême. Les viandes proscrites excitaient alors moins l'appétit que les plats de poissons servis aux tables des grands seigneurs, qui permettaient de se régaler tout en restant un bon chrétien.

\section{Le hareng et l'anguille dans la branche III du Roman de Renart}

Cependant, des poissons plus communs suscitent aussi les convoitises dans les œuvres comiques, notamment dans le Roman de Renart, où le désir de nourriture occupe une place primordiale, comme l'a souligné Jacques Le Goff : «Théâtre, épopée de la faim, il nous montre Renart, sa famille, ses compagnons, sans cesse mus par l'appel de leur ventre creux. Le ressort de presque toutes les «branches » du cycle, c'est la faim omniprésente et omnipotente - mobile de la

\footnotetext{
${ }^{15}$ «Les Trois Dames qui troverent l'anel », Nouveau Recueil Complet des Fabliaux, op. cit., tome II, 11b, p. 232, v. 116 sqq.

16 Douin de lavesne, Trubert, éd. Guy Raynaud De Lage, Genève, Droz, 1974, p. 93, v. 2802.
} 
ruse de Renart ${ }^{17}$. » Ainsi, dans la première partie de la branche III, le goupil affamé déploie des trésors d'ingéniosité pour manger des harengs et des anguilles. Pourquoi ces deux espèces ? Des travaux d'archéozoologie ont montré que ces deux poissons occupaient une place prépondérante dans la consommation du Nord de France ${ }^{18}$. Le passage des charrettes de harengs destinées à l'approvisionnement des grandes villes était un spectacle familier aux habitants de ces régions. Toutefois, le hareng et l'anguille ne sont pas choisis pour de simples raisons de réalisme, mais parce qu'ils mettent en jeu des codes alimentaires spécifiques et propres à la période médiévale.

La faim est bien, comme dans l'ensemble du cycle, le principal ressort dramatique de cette branche. Dès les premiers vers, elle est placée au cœur du récit, qui s'ouvre sur une "anti-reverdie »: au lieu de commencer à la belle saison, sur le tableau d'un retour à la vie et à la joie, l'action s'engage à l'approche de l'hiver sous le signe d'une douleur profonde causée par la faim. Cette dernière est dite au moyen de la négation ; elle se traduit par l'impuissance de Renart et s'exprime physiquement par une agitation frénétique ${ }^{19}$ à laquelle succède la passivité de l'attente. Renart est totalement dominé par son désir animal. L'espoir qui lance l'aventure vient de la nourriture de saison: les poissons. L'abondance succède alors brutalement à la stérilité. Renart reprend sur-le-champ la maîtrise sur lui-même et sur les événements. L'animal aux abois redevient «Renart qui tot siecle abeite » ${ }^{20}$. Comme le suggère le sens étymologique du verbe abeiter (« faire mordre à l'hameçon, appâter »), Renart se change en pêcheur à la vue de sa proie et au mouvement désordonné succède une trajectoire inexorable vers l'objectif, comparable à celle d'une flèche. Au règne de la faim succède le temps de la ruse. Lorsque Renart repu rentre à son

\footnotetext{
${ }^{17}$ Jacques Le Goff, La Civilisation de l'Occident médiéval, Paris, Arthaud, 1964, p. 292.

${ }^{18}$ Benoît Clavel, L'Animal dans l'alimentation médiévale et moderne en France du Nord (XII ${ }^{e}-X V I I^{e}$ siècles), Revue archéologique de Picardie (nº spécial 19), 2001, p. 160.

${ }^{19}$ Le Roman de Renart, op. cit., p. 280, v. 16 : «Molt coloie de totes parz. »

${ }^{20}$ Ibid., p. 282, v. 34.
} 
château, le contentement a remplacé la détresse. Sa démarche est sautillante et joyeuse $^{21}$.

Intéressons-nous maintenant aux aliments qui ont rendu possible cette spectaculaire métamorphose. L'appétit de Renart pour le hareng peut surprendre car c'est un poisson tout à fait ordinaire. Certains textes littéraires expriment même une lassitude par rapport au sempiternel hareng salé des jours maigres. Ainsi à la fin du XIV $\mathrm{XI}^{\mathrm{e}}$ siècle, dans sa ballade 350 contre le carême, Eustache Deschamps stigmatise les «harens puanz, poissons de mer pourris ${ }^{22} »$. Les proverbes évoquent souvent la puanteur du hareng, comme celui qui est rapporté dans une parodie hagiographique de la fin $X V^{\mathrm{e}}$ siècle, le «Sermon joyeux de saint Hareng » :

Se harenc put, c'est sa nature,

S'il fleurre bon, c'est adventure. ${ }^{23}$

Mais le poète précise immédiatement que les pauvres gens ne tiennent pas ce discours car le hareng leur sauve souvent la vie ${ }^{24}$. Le texte insiste plutôt sur les miracles qu'il accomplit. En période d'abstinence, le hareng permet d'affronter les privations, c'est une nourriture providentielle et saine qui sauve les hommes médiévaux de la faim. Sa dévalorisation intervient assez tard. La formidable explosion du commerce du hareng salé au cours du XIII ${ }^{\mathrm{e}}$ siècle a fait de cet aliment providentiel un mets ordinaire, jusqu'à l'écœurement. La littérature semble enregistrer ce dégoût à partir de la fin du XIV ${ }^{\mathrm{e}}$ siècle, mais le hareng est au contraire valorisé à l'extrême dans les textes antérieurs.

Le hareng n'est pas seulement un poisson de pauvre : on peut offrir sans rougir des harengs frais aux grands personnages. Ils figurent d'ailleurs dans la

\footnotetext{
${ }^{21}$ Ibid., p. 288, v. 158-161 : «Cil saillirent contre leur pere, / Qui s'en venoit les menus saus, /Gros et saoulz, joieus et baus, / Les anguilles entour son col. »

${ }_{22}^{22}$ Eustache Deschamps, Oeuvres complètes, éd. Marquis QueuX DE SAINT-HILAIRE, Paris, Firmin-Didot, 1882, tome III, ballade CCCL, v. 22.

${ }^{23}$ «Sermon joyeux de saint Hareng », Recueil de sermons joyeux, éd. Jelle Koopmans, Genève, Droz, 1988 (coll. TLF, 362), v. 98-99.

${ }^{24}$ Ibid., v. 100-101 : « Povres gens ne le dient mie/ Car souvent leur sauve la vie. »
} 
liste du Roman du Comte d'Anjou. Même lorsqu'il est salé, c'est un produit apprécié au marché. Entendons par exemple ce cri de la marchande de harengs saurs :

La Crieresse d'Arens :

Harens soretz appetissants

Ce sont petis morceaux frians

Pour desjuner au matinet

Avec vin blanc, cler, pur et net. ${ }^{25}$

De plus, on nommait par métonymie « appétit », le hareng bouffi, une variété de hareng saur, parce qu'il avait la réputation d'ouvrir l'appétit. Le hareng est aussi le poisson idéal des savants. Cosmologies et ouvrages de médecine font son apologie. Pour les médecins, très circonspects à l'égard du poisson en général, il réunit toutes les qualités : c'est un poisson de mer fusiforme, couvert d'écailles, qui se déplace et absorbe une nourriture pure. On lui attribue de nombreuses vertus thérapeutiques, en particulier des propriétés désinfectantes. Dans la plupart des encyclopédies, c'est même un animal mythique : le hareng est l'animal qui se nourrit exclusivement d'eau de mer selon le Placides et Timeo, Barthélémy l'Anglais ou encore Vincent de Beauvais. Même les savants qui réfutent cette affirmation, comme Albert le Grand ${ }^{26}$, prêtent au hareng des qualités diététiques et thérapeutiques qui accréditent l'image d'un poisson spécialement sain.

Il est par excellence le poisson qui transmet à l'homme les vertus nutritives et purifiantes de la mer; à ce titre, il n'exige pas autant d'apprêt, de préparation culinaire que les autres espèces. Ses propriétés n'ont pas besoin d'être amendées. Le fait que Renart mange les harengs crus et sans assaisonnement est certainement un indice de bestialité. Le Roman de Renart joue sur un va-et-vient incessant entre représentations anthropomorphiques et ${ }^{25}$ Cris de Paris, Paris, BnF, Arsenal, Est. 264 Rés. Ce recueil est illustré de gravures sur bois coloriées réalisées vers 1500 .

${ }^{26}$ AlBert le Grand, De Animalibus, éd. Hermann Stadler, Münster, 1916-1920, XXIV, 8, p. 1518. Cette croyance tient selon lui à l'absence d'intestins dans le ventre du hareng. 
zoomorphiques et sur les ambiguités ainsi produites. Renart dévore le hareng comme un animal alors qu'il déguste les anguilles comme un seigneur humain. Toutefois le texte insiste sur l'absence d'assaisonnement et non sur la crudité :

Mout par en menja volentiers, Onques n'i quist ne sel ne sauge. ${ }^{27}$

On peut alors proposer une autre interprétation de cette dévoration du hareng «nature »: Renart s'approprie directement cette nourriture primordiale qui recèle une force vitale venue de la mer et que n'ont pas les autres poissons. Dans la suite de la branche, ragaillardi par ce hors-d'œuvre, il déploie d'ailleurs une énergie et une ruse hors du commun.

Mais le poisson qui tient le premier rôle est sans conteste l'anguille. Elle fait le lien entre les deux épisodes de la branche : le vol des poissons et la pêche à la queue. En effet, pour piéger Ysengrin, Renart l'appâte en lui faisant goûter de petits morceaux des anguilles dérobées aux marchands. L'anguille assure donc la cohérence de la narration, en lui permettant de rebondir alors que les appétits de Renart et sa famille ont été comblés. Pourquoi cet épisode ne pouvait-il être développé autour d'une autre espèce ? Les harengs suffisent à calmer la faim; le vol des anguilles est commandé par la gourmandise. L'anguille est l'une des cibles privilégiées de la gula $^{28}$, le «péché de bouche », qui est associé au «péché de chair», à la luxure ${ }^{29}$. L'aliment qui évoque le mieux la réunion de ces deux péchés, c'est évidemment le poisson-serpent, celui-là même qui reçoit son nom de cette ressemblance. Selon une étymologie d'Isidore reprise par tous les encyclopédistes, l'anguilla tire son nom de l'anguis $^{30}$. De nombreux autres indices font d'elle un être hybride et donc un

\footnotetext{
${ }^{27}$ Le Roman de Renart, op. cit., p. 284, v. 88-89.

${ }^{28} \mathrm{La}$ traduction par le terme de « gourmandise » n'est pas entièrement satisfaisante. La gula, littéralement le « péché de gueule », est à la fois recherche de la quantité et du raffinement.

${ }^{29}$ Les théologiens du Moyen Âge se demandent par exemple si le péché originel n'est pas la gula. Même si elle est contestée par saint Thomas d'Aquin, cette opinion, favorisée par une lecture littérale de la Bible, est encore courante au XIII ${ }^{\mathrm{e}}$ siècle.

${ }^{30}$ IsIDORE DE SÉVILLE, Etymologiae, XII, éd. J. André, Paris, Les Belles Lettres, 1986, p. 205.
} 
aliment suspect : elle naît par génération spontanée de la boue ou des déchets produits par les autres poissons ; elle peut vivre jusqu'à six jours hors de l'eau et son cadavre, contrairement à celui des autres poissons, ne flotte pas avant de se désagréger dans l'eau.

Fortement représentée dans les livres de cuisine, l'anguille est fermement déconseillée par les savants. Même s'ils ne cherchent pas à dissuader leurs lecteurs de consommer de l'anguille - peut-être est-ce une habitude trop bien ancrée dans les pratiques médiévales -, ils donnent à défaut des conseils pour réduire sa nocivité : ils recommandent, après l'avoir écorchée, de la cuire plus que les autres poissons et de la faire rôtir car c'est le mode de cuisson qui dessèche le plus. Cela lui ôte son humidité excessive, et ainsi, «sa mauvaise nature s'évapore $\rangle^{31}$. Hildegarde de Bingen quant à elle la condamne sans appel : «Même si elle ne cherche pas particulièrement les aliments immondes, sa chair a quelque chose d'impur comme celle du porc. Elle accable les malades au cours de leurs fièvres, dans leurs épanchements d'humeurs et dans toutes les maladies. À ceux qui la mangent, elle donne un esprit rempli d'amertume et elle les rend retors et soupçonneux ${ }^{32}$.» L'adjectif astutos appliqué par l'abbesse aux mangeurs d'anguille renvoie bien sûr ici à une ruse malveillante voire diabolique. La littérature médiévale semble confirmer cette remarque, car l'anguille présente des affinités avec les personnages rusés et en particulier avec Renart. Nous ne développerons pas ici ce point qui s'écarte du sujet de la faim et de l'appétit, mais il explique aussi l'importance des anguilles dans cette branche. En dérobant les anguilles pour les déguster en famille, Renart obéit à un appétit

\footnotetext{
${ }^{31}$ Thomas de CANTIMPRÉ, Liber de natura rerum, éd. Helmut Boese, Berlin-New York, 1973, VII, 2, p. 253. Voir aussi VIncent DE BeAuvaIS, Speculum majus, t. I, Douai, 1624, reprint Graz, 1965, XVII, 31, col. 1270 et AlBert le GrAND, De Animalibus, op. cit., vol. 2, XXIV, 4, p. 1519.

${ }^{32}$ Hildegarde De Bingen, Le Livre des subtilités des créatures divines, trad. Pierre Monat, Grenoble, Jérôme Millon, 1989, p. 119. Pour le texte latin, voir HiLdEGARDE DE BingEN, Sanctae Hildegardis abbatissae subtilitatum diversarum naturarum creaturarum libri novem (Physica), Patrologiae cursus completus, series latina, éd. Jacques-Paul Migne, Turnhout, Brepols, 1991 (réimp. de l'éd. de Paris, 1855), vol. 197, V, 33, col. 1283-1284.
} 
coupable. Sa ruse extraordinaire le met pourtant à l'abri de tout châtiment. Seul le loup sera puni à la fin de la branche, moins pour son avidité que pour son cruel manque d'astuce.

Les appétits des hommes du Moyen Âge nous sont en partie étrangers. L'interprétation des mentions alimentaires dans les textes littéraires médiévaux sollicite une attention vigilante aux codes divers qui les déterminent : le réseau narratif et symbolique propre à chaque œuvre littéraire, mais aussi les contextes matériels et culturels auxquels elles se rattachent. L'attrait particulièrement vif pour les poissons dans la littérature médiévale fait certainement écho à des frustrations ressenties par les chrétiens du Moyen Âge, astreints à de longues périodes de jeûne pendant lesquelles des poissons variés et frais constituaient la plus grande richesse imaginable. La gourmandise, lorsqu'elle se porte sur le poisson est encore plus scandaleuse, car elle pervertit le sens de l'abstinence chrétienne. Par ailleurs, alors que les mentions du poisson en général dans les textes littéraires sont relativement peu significatives, un imaginaire riche et varié est mis en jeu lorsque des espèces précises sont évoquées. Les différentes espèces de poissons dessinent une hiérarchie sociale, et les représentations scientifiques, notamment médicales, nous permettent d'envisager des significations qui ne sont pas évidentes pour un lecteur moderne. Le hareng est une nourriture providentielle, accessible à tous, qui calme la faim et revigore les corps ; l'anguille est le poisson du péché, du plaisir des sens, mais aussi de la ruse ; les poissons royaux connotent le luxe et l'abondance. La sélection de ces espèces merveilleuses - le brochet, la lamproie, le saumon - mériterait également que l'on s'y intéresse : chacune renvoie à des univers ou des codes différents. Le choix des nourritures qui excitent la faim et l'appétit des personnages littéraires du Moyen Âge n'est jamais anodin. 